\section{Efecto de una intervención centrada en la reducción de factores de riesgo cardiovascular en estudiantes universitarios}

\author{
ANA MARÍA LEIVA O. ${ }^{1, a}$, MARÍA ADELA MARTÍNEZ S., ${ }^{2, b}$, \\ CARLOS CELIS-MORALES ${ }^{3, c}$
}

\section{Effects of an intervention to reduce cardiovascular risk factors in university students}

Background: Interventions aiming to reduce obesity and sedentary behaviors in young adults could be a feasible and effective approach to prevent cardiovascular diseases. Aim: To evaluate the effect of a lifestyle-based intervention on reducing cardiovascular risk factors in university students. Material and Methods: Sixty university students aged $21 \pm 1$ years $(n=44$ females) took part on a 17 weeks lifestyle intervention consisting in education about healthy lifestyles and physical training, during a curricular course about health promotion and healthy lifestyles. At baseline and at the end of the intervention participants completed a lifestyles questionnaire and provided fasting blood samples to quantify glucose and lipids profile. Results: After the intervention significant reductions in the prevalence of hyperglycemia (-10.0\%), high blood pressure (-16.7\%) and physical inactivity (-26.7\%) were observed. Moreover, a significant reduction in body weight, waist circumference, blood pressure, total cholesterol, triglycerides and an increase in HDL cholesterol were observed after intervention. Conclusions: The implementation of an intervention to promote healthy lifestyles is an effective way of reducing cardiovascular risk factors in university students.

(Rev Med Chile 2015; 143: 971-978)

Key words: Interventions studies; Life style; Motor activity; Obesity.

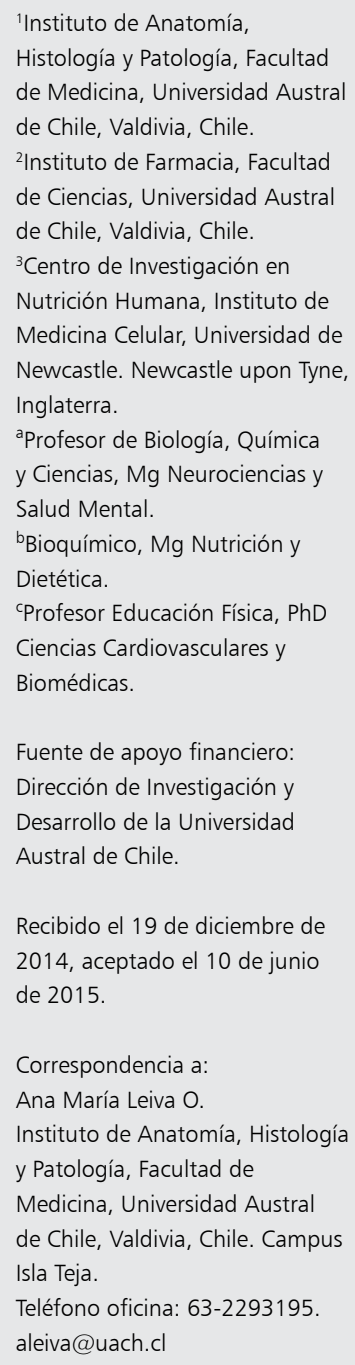

Fuente de apoyo financiero: Dirección de Investigación y Desarrollo de la Universidad Austral de Chile.

Recibido el 19 de diciembre de 2014, aceptado el 10 de junio de 2015.

Correspondencia a: Ana María Leiva O. Instituto de Anatomía, Histología y Patología, Facultad de Medicina, Universidad Austral de Chile, Valdivia, Chile. Campus Isla Teja.

Teléfono oficina: 63-2293195 aleiva@uach.cl

$\mathrm{E}$ n la actualidad, existe preocupación por el alarmante aumento de las enfermedades crónicas no transmisibles; dentro de este grupo, las enfermedades cardiovasculares (ECV) siguen siendo la principal causa de morbimortalidad a nivel mundial ${ }^{1}$.

Si bien la etiología de las ECV es multifactorial, existen factores de riesgo para estas enfermedades susceptibles de ser modificados; siendo la promoción de la salud y la adopción de estilos de vida saludable, aspectos claves en su prevención ${ }^{1,2}$.

En Chile, las ECV representan la principal causa de muerte de la población, con una alta prevalencia de sus principales factores de riesgo, los que se manifiestan con desigualdades según edad, sexo y nivel educacional ${ }^{3}$. Esta situación ha significado que su prevención y control sean uno de los principales desafíos sanitarios del país; implementándose diversas iniciativas de carácter intersectorial y participativo, que apuntan a promover estilos de vida saludable en la población ${ }^{4,5}$.

Como una forma de conocer la realidad local de los jóvenes respecto a sus factores de riesgo para estas enfermedades, el año 2007, se realizó 
Intervención en factores de riesgo cardiovascular en estudiantes - A. M. Leiva et al

una investigación en estudiantes de primer año de la Universidad Austral de Chile (UACh), que evidenció estilos de vida poco saludables asociados a los principales factores de riesgo cardiovascular $(\text { FRCV })^{6}$. Frente a esta realidad, y con el fin de encontrar una estrategia que permita la adopción de estilos de vida saludable por parte de los jóvenes, se evaluó el efecto de una intervención centrada en la reducción de FRCV en estudiantes de la UACh, en el marco de una asignatura de promoción de la salud.

\section{Material y Método}

\section{Diseño y sujetos de estudio}

Estudio cuasi-experimental centrado en la reducción de FRCV en estudiantes que cursan tercer año en la UACh, a partir de una asignatura optativa dictada el segundo semestre del año 2011. El tamaño de la muestra quedó condicionado al número de cupos de la asignatura. La muestra intencionada quedó conformada por 60 estudiantes, quienes tras inscribir la asignatura firmaron un consentimiento informado y una carta compromiso individual en relación a su autocuidado.

La asignatura "Promoción de salud y estilos de vida saludable" se ajustó a un semestre académico, con 17 semanas. La etapa de intervención consideró dos ejes de trabajo: Educación para la salud y Actividad física (Tabla 1).

El eje "Educación para la salud" estuvo a cargo de académicos especialistas en las materias abordadas. Las clases teóricas de $45 \mathrm{~min}$ de duración alternaron talleres y consejerías en alimentación y estilos de vida saludable. El eje "Actividad física" estuvo a cargo de estudiantes egresados de la carrera de Educación Física y Salud. Se ofertaron tres sesiones semanales de actividades deportivo-recreativas, distribuidas en 17 semanas, con una duración de 60 min cada una. El requisito para la aprobación de la asignatura fue asistir a dos de las tres sesiones programadas semanalmente. El detalle de las actividades desarrolladas en la asignatura se muestra en la Tabla 2.

\section{Recolección de la información}

Durante la primera semana de la asignatura (etapa de pre-intervención) se realizó el diagnóstico y en la última semana (etapa de post-intervención), la evaluación final. En ambas etapas se citó a los estudiantes en ayunas con el fin de extraer una muestra de sangre para determinar glicemia y perfil lipídico. Se tomaron medidas antropométricas, se midió la presión arterial y se aplicó un cuestionario, validado en estudio anterior que consideró antecedentes sociodemográficos y de estilos de vida ${ }^{6}$.

\section{Variables en estudio}

Las variables sociodemográficas consideraron edad, sexo, residencia, área de estudio y nivel socioeconómico.

Los parámetros estudiados fueron glicemia, colesterol total (CT), triglicéridos (TG), colesterol LDL (cLDL), colesterol HDL (cHDL) y presión arterial (PA). Los puntos de corte para cada uno de estos parámetros se definieron en base a los criterios utilizados en la segunda Encuesta $\mathrm{Na}-$ cional de Salud 2009-2010 (ENS 2009-2010) ${ }^{3}$. Se consideró glicemia elevada, valores $\geq 100 \mathrm{mg} / \mathrm{dL}$ y posible diabetes mellitus no confirmada a valores de glicemia $\geq 126 \mathrm{mg} / \mathrm{dL}$. Los puntos de corte para parámetros alterados del perfil lipídico fueron: $\mathrm{CT} \geq 200 \mathrm{mg} / \mathrm{dL}, \mathrm{cLDL} \geq 130 \mathrm{mg} / \mathrm{dL}, \mathrm{TG} \geq 150$ $\mathrm{mg} / \mathrm{dL}$ y valores bajos de cHDL $<40 \mathrm{mg} / \mathrm{dL}$. Se consideró estado de pre-hipertensión, valores de PA sistólica (PAS) 120-139 mmHg o PA diastólica (PAD) 80-89 mmHg; y un estado de hipertensión arterial (HTA) valores de PAS $\geq 140 \mathrm{mmHg}$ o PAD $\geq 90 \mathrm{mmHg}$.

Las medidas antropométricas incluyeron índice de masa corporal (IMC) y circunferencia cintura (CC). Los protocolos de estas mediciones

Tabla 1. Etapas de la asignatura "Promoción de salud y estilos de vida saludable"

\begin{tabular}{|lll|}
\hline Pre-intervención & Intervención & Post-intervención \\
\hline Recolección inicial de información & Ejes de trabajo & Recolección final de información \\
$\begin{array}{l}\text { - Medición de parámetros biofísicos } \\
\text { - Aplicación de cuestionario de estilos } \\
\text { de vida }\end{array}$ & - Educación para la salud & - Medición de parámetros biofísicos \\
\hline
\end{tabular}


Tabla 2. Ejes de intervención, objetivos y actividades desarrolladas en la asignatura: "Promoción de salud y estilos de vida saludable"

\begin{tabular}{|c|c|c|}
\hline Ejes de intervención & Objetivos & Actividades \\
\hline Educación para la salud & $\begin{array}{l}\text { Promover la adopción } \\
\text { de estilos de vida } \\
\text { saludable }\end{array}$ & $\begin{array}{l}\text { Clases teóricas: } 5 \text { sesiones de } 45 \text { min c/u } \\
\text { - Introducción a la asignatura, firma de consentimiento informado y } \\
\text { compromiso individual con su autocuidado. Aplicación de cuestio- } \\
\text { nario estilos de vida } \\
\text { - } \quad \text { Estilos de vida y factores de riesgo cardiovascular } \\
\text { - } \text { Beneficios de la actividad física para la salud } \\
\text { - } \quad \text { Alimentación saludable } \\
\text { - Guías alimentarias y etiquetado nutricional } \\
\text { Talleres prácticos: } 2 \text { sesiones de } 90 \text { min c/u } \\
\text { - Degustación comida saludable (con sus recetas) } \\
\text { - } \quad \text { Aprendiendo a cocinar saludable } \\
\text { Consejerías: } 2 \text { sesiones de } 45 \text { min c/u } \\
\text { - Alimentación saludable } \\
\text { - Plan de actividad física según necesidades }\end{array}$ \\
\hline Actividad física & $\begin{array}{l}\text { Incentivar la práctica } \\
\text { regular de actividad } \\
\text { física }\end{array}$ & $\begin{array}{l}\text { Actividades deportivo-recreativas: } 3 \text { sesiones semanales de } 60 \text { min c/u, } \\
\text { distribuidas en } 17 \text { semanas } \\
\text { - Caminata rápida, baile entretenido, trote, kayak, acondicionamien- } \\
\text { to físico, futbol, zumba, voleibol, juegos al aire libre }\end{array}$ \\
\hline
\end{tabular}

y sus puntos de corte se basaron en la ENS 2009$2010^{3}$. Se consideró exceso de peso a un IMC $\geq 25$ $\mathrm{kg} / \mathrm{m}^{2}$, sobrepeso a un IMC: $25-29,9 \mathrm{~kg} / \mathrm{m}^{2}$ y un IMC $\geq 30 \mathrm{~kg} / \mathrm{m}^{2}$ como obesidad. Para diagnosticar obesidad abdominal se utilizó el criterio validado para la población chilena $\mathrm{CC} \geq 88 \mathrm{~cm}$ para varones, $C C \geq 83 \mathrm{~cm}$ para mujeres.

El cuestionario de estilos de vida evaluó la actividad física a través de dos preguntas, la primera relacionada con la frecuencia de realización de ejercicio físico o deporte, dando como opciones "3 o más veces por semana", " $1-2$ veces por semana", "1-3 veces por mes" y "Nunca”. Se consideró sedentario al estudiante que respondió no practicar actividad física 3 o más veces por semana. La segunda pregunta indaga sobre las barreras que le impiden practicar actividad física, considerando las siguientes opciones: "No tengo tiempo", "No estoy acostumbrado(a)", "Prefiero ver televisión o usar computador" y "No me gusta".

El consumo de frutas y verduras se evaluó a partir de 2 preguntas relacionadas con la frecuencia y cantidad. La frecuencia se evaluó con las siguientes opciones: "Todos los días", "4-6 días a la semana", "2-3 días a la semana", "1 día a la semana” y "1-2 veces al mes". La cantidad se evaluó de acuerdo al número de porciones consumidas, a partir de una pregunta abierta. Se consideró bajo consumo de frutas y verduras, a quienes declararon consumir menos de 5 porciones diarias de estos alimentos ${ }^{3}$.

El consumo de tabaco se evaluó considerando "fumador actual" al estudiante que señaló fumar, distinguiéndose entre ellos al "fumador diario": al menos un cigarrillo/día y "fumador ocasional": menos de un cigarrillo/día ${ }^{3}$.

El consumo de alcohol se evaluó según la frecuencia de consumo durante la semana y la cantidad de tragos consumidos en un día habitual. Se consideró bebedor moderado al estudiante que señaló consumir 1-2 tragos, bebedor en riesgo al que indicó consumir 3-5 tragos y bebedor excesivo si bebe más de 5 tragos en una sola ocasión ${ }^{7}$.

\section{Análisis de datos}

Los resultados son presentados como media y su respectiva desviación estándar para variables de tipo continua. La distribución normal de las variables fue verificada con el test de Anderson-Darling. Para determinar si los cambios en las variables de tipo continuas fueron significativos entre pre y post-intervención se aplicó $t$-test para 
muestras pareadas. Para variables categóricas, los resultados fueron presentados como prevalencia. Para determinar si los cambios en prevalencia pre y post-intervención fueron significativos, se aplicó el test $\chi^{2}$.

\section{Medidas básicas para la valoración de resultados}

Para evaluar la efectividad de la intervención se consideró las recomendaciones de la Organización Mundial de la Salud (OMS) ${ }^{8}$, considerando cambios de estilos de vida (hábitos alimentarios, frecuencia de actividad física, consumo de alcohol y tabaco) y cambios biofísicos (glicemia, perfil lipídico, presión arterial y medidas antropométricas).

Este estudio fue aprobado por el Comité de Ética de la UACh.

\section{Resultados}

De la muestra intencionada de 60 estudiantes, $73 \%$ correspondió a mujeres. La edad promedio fue 20,7 $\pm 0,9$ años para mujeres y 20,7 $\pm 1,4$ años para hombres. En cuanto a su residencia, $40 \%$ residía en cabañas, $36,7 \%$ vivía con su familia, $15 \%$ residía en pensiones y $8,3 \%$ en hogares estudiantiles. En relación a sus áreas de estudio, $55 \%$ de ellos cursaba carreras de la salud, 30\% ciencias biológicas, $8,3 \%$ filosofía y humanidades, y $6,7 \%$ ingenierías. Respecto a la caracterización socio-económica, $58,3 \%$ de los estudiantes pertenecía a los tres quintiles de menor ingreso, según información del ingreso autónomo per-cápita del hogar?.

El diagnóstico realizado en la población estudiada en la etapa de pre-intervención encontró una alta prevalencia de todos los FRCV examinados. Al analizar las prevalencias según sexo, se observó que las mujeres eran las que presentaban los mayores porcentajes de FRCV, sin embargo, esta diferencia no fue significativa. Al explorar si existía asociación entre la prevalencia de estos FRCV y exceso de peso, se encontró una asociación significativa con glicemia alterada $(\mathrm{p}=0,001)$ y sedentarismo $(p=0,001)$. No se encontró relación con CT elevado, cLDL elevado, TG elevado, cHDL bajo y PA elevada.

Las prevalencias de los principales FRCV, comparando etapas pre y post-intervención se presentan en la Tabla 3. Se destaca que la intervención favoreció la reducción de las prevalencias de todos los FRCV en la población estudiada, siendo estos cambios significativos para sedentarismo $(-26,7 \%)$, glicemia elevada $(-10,0 \%)$ y PA elevada $(-16,7 \%)$. Al comparar las prevalencias según sexo; se aprecia que la intervención favoreció la reducción de la mayor parte de los FRCV, con resultados significativos para PA elevada en mujeres $\mathrm{y}$ sedentarismo en hombres.

Tabla 3. Prevalencias de factores de riesgo cardiovascular en etapas pre y post-intervención, según sexo

\begin{tabular}{|lccccccccc|}
\hline $\begin{array}{l}\text { Factores riesgo } \\
\text { cardiovascular }\end{array}$ & \multicolumn{3}{c}{ Mujeres \% } & \multicolumn{3}{c}{ Hombres \% } & \multicolumn{3}{c|}{ Total \% } \\
\hline Exceso de peso & Prest & Valor $\mathbf{p}$ & Pre & Post & Valor $\mathbf{p}$ & Pre & Post & Valor p \\
\hline Obesidad abdominal & 45,5 & 34,1 & 0,281 & 37,5 & 18,8 & 0,252 & 43,3 & 30,0 & 0,131 \\
\hline Colesterol total elevado & 36,4 & 27,3 & 0,365 & 31,3 & 18,8 & 0,430 & 35,0 & 25,0 & 0,235 \\
\hline Colesterol HDL bajo & 56,8 & 38,6 & 0,089 & 18,8 & 6,3 & 0,300 & 46,7 & 30,0 & 0,061 \\
\hline Colesterol LDL elevado & 27,3 & 11,3 & 0,059 & 18,8 & 18,8 & 1,000 & 25,0 & 13,3 & 0,106 \\
\hline Triglicéridos elevados & 34,1 & 31,8 & 0,823 & 31,2 & 25,0 & 0,705 & 33,3 & 30,0 & 0,697 \\
\hline Glicemia elevada & 25,0 & 22,7 & 0,487 & 12,5 & 0,0 & 0,472 & 21,7 & 16,7 & 0,490 \\
\hline Presión arterial elevada & 15,9 & 4,5 & 0,080 & 6,3 & 0,0 & 0,325 & 13,3 & 3,3 & $\mathbf{0 , 0 4 8}$ \\
\hline Sedentarismo & 15,9 & 0,0 & $\mathbf{0 , 0 0 5}$ & 18,8 & 0,0 & 0,072 & 16,7 & 0,0 & $\mathbf{0 , 0 0 0 8}$ \\
\hline Tabaquismo & 81,8 & 63,6 & 0,056 & 75,0 & 25,0 & $\mathbf{0 , 0 0 3}$ & 80,0 & 53,3 & $\mathbf{0 , 0 0 1}$ \\
\hline Consumo riesgoso de alcohol & 52,3 & 36,4 & 0,136 & 43,7 & 43,7 & 1,000 & 50,0 & 38,4 & 0,201 \\
\hline
\end{tabular}

Pre: Pre-intervención; Post: Post-intervención. 
Tabla 4. Descripción de las variables antropométricas y metabólicas pre y post-intervención, según sexo

\begin{tabular}{|c|c|c|c|c|c|c|c|c|c|}
\hline & & Mujeres & & & mbres & & & Total & \\
\hline & Pre & Post & Valor p & Pre & Post & Valor $\mathbf{p}$ & Pre & Post & Valor $p$ \\
\hline Peso corporal $(\mathrm{kg})$ & $65,8 \pm 11,0$ & $63,6 \pm 9,4$ & $<0,0001$ & $73,0 \pm 8,6$ & $71,1 \pm 6,8$ & 0,004 & $67,7 \pm 10,8$ & $65,6 \pm 9,3$ & $<0,0001$ \\
\hline IMC $\left(\mathrm{kg} / \mathrm{m}^{2}\right)$ & $24,9 \pm 4,2$ & $24,0 \pm 3,6$ & $<0,0001$ & $24,4 \pm 2,7$ & $23,7 \pm 2,3$ & 0,003 & $24,8 \pm 3,9$ & $23,9 \pm 3,3$ & $<0,0001$ \\
\hline $\mathrm{CC}(\mathrm{cm})$ & $78,2 \pm 10,1$ & $79,2 \pm 12,0$ & 0,0005 & $83,5 \pm 5,2$ & $82,9 \pm 4,8$ & 0,278 & $79,6 \pm 9,3$ & $80,3 \pm 9,91$ & 0,020 \\
\hline Glicemia (mg/dL) & $93,2 \pm 6,5$ & $91,9 \pm 4,9$ & 0,107 & $88,7 \pm 8,0$ & $91,4 \pm 5,7$ & 0,135 & $92,0 \pm 7,1$ & $91,8 \pm 5,1$ & 0,789 \\
\hline PAD $(\mathrm{mmHg})$ & $68,6 \pm 11,2$ & $70,6 \pm 4,1$ & 0,225 & $69,8 \pm 10,2$ & $72,0 \pm 4,7$ & 0,523 & $69,0 \pm 10,9$ & $70,7 \pm 4,2$ & 0,168 \\
\hline PAS $(\mathrm{mmHg})$ & $102,5 \pm 12,0$ & $97,9 \pm 8,1$ & 0,002 & $104,9 \pm 8,9$ & $98,2 \pm 9,0$ & 0,007 & $103,2 \pm 11,2$ & $98,0 \pm 8,3$ & $<0,0001$ \\
\hline$C T(\mathrm{mg} / \mathrm{dL})$ & $206,1 \pm 43,3$ & $185,8 \pm 29,9$ & $<0,0001$ & $161,8 \pm 29,0$ & $157,2 \pm 25,5$ & 0,470 & $194,3 \pm 44,4$ & $178,2 \pm 31,3$ & $<0,0001$ \\
\hline $\mathrm{TG}(\mathrm{mg} / \mathrm{dL})$ & $134,0 \pm 69,9$ & $117,0 \pm 41,2$ & 0,003 & $93,0 \pm 14,3$ & $92,0 \pm 14,54$ & 0,469 & $123,1 \pm 62,9$ & $110,1 \pm 37,7$ & 0,003 \\
\hline $\mathrm{cHDL}(\mathrm{mg} / \mathrm{dL})$ & $48,9 \pm 12,4$ & $53,4 \pm 10,7$ & $<0,0001$ & $51,0 \pm 8,9$ & $53,8 \pm 9,9$ & 0,004 & $48,7 \pm 11,5$ & $53,5 \pm 10,4$ & $<0,0001$ \\
\hline $\mathrm{cLDL}(\mathrm{mg} / \mathrm{dL})$ & $110,6 \pm 35,4$ & $110,5 \pm 35,3$ & 0,197 & $119,8 \pm 23,8$ & $118,7 \pm 22,5$ & 0,469 & $113,4 \pm 32,7$ & $112,7 \pm 32,4$ & 0,158 \\
\hline
\end{tabular}

Datos presentados como Media y Desviación Estándar. Diferencias entre Pre y Post-intervención fueron analizadas mediante t-test para muestras pareadas. Pre: pre-intervención; Post: post-intervención; IMC: índice masa corporal; CC: circunferencia cintura; PAD: promedio presión arterial diastólica; PAS: promedio presión arterial sistólica; CT: colesterol total; TG: triglicéridos; cHDL: colesterol HDL; $c L D L$ : colesterol LDL.

En cuanto al consumo de alcohol en la etapa de pre-intervención, $81,7 \%$ de los estudiantes declaró consumir alcohol, siendo 50\% bebedor en riesgo, concentrando el consumo en un día a la semana. En la etapa de post-intervención no hubo variación en el porcentaje de los estudiantes que bebían, pero sí en la cantidad de tragos que declararon consumir en una oportunidad, disminuyendo a $38,4 \%$ los bebedores en riesgo (Tabla 3 ).

En la Tabla 4 se presenta la descripción de las variables antropométricas y metabólicas pre y post-intervención. Se observa que posterior a la intervención se redujo significativamente para ambos sexos, el peso corporal, el IMC, la PAS y aumentó significativamente el cHDL. Las variables CC, CT y TG sólo disminuyeron significativamente en mujeres. Otros marcadores de salud metabólica como glicemia basal, PAD y cLDL, no presentaron cambios significativos en la población estudiada.

En relación al consumo de frutas y verduras en la etapa de pre-intervención, todos los estudiantes declararon tener un bajo consumo este tipo de alimentos. $\mathrm{Al}$ analizar la frecuencia de consumo, $21,7 \%$ consumía frutas y verduras todos los días, $45 \% 2-3$ días a la semana, $28,3 \%$ un día a la semana y $5 \%$ una a dos veces al mes. Al considerar la cantidad de frutas y verduras consumidas, ningún es- tudiante cumplía con la recomendación de 5 o más porciones diarias de frutas y verduras. En la etapa de post-intervención, el consumo diario de frutas y verduras aumentó a 55\%, declarando 20\% de los estudiantes consumir 5 o más porciones diarias, logrando así cumplir con las recomendaciones.

\section{Discusión}

El objetivo de este trabajo fue evaluar el efecto de una intervención centrada en la reducción de FRCV en estudiantes universitarios, evidenciándose una mejora significativa en marcadores de obesidad y metabólicos y una disminución en las prevalencias de PA elevada, glicemia elevada y sedentarismo.

Considerando que la población en estudio fueron jóvenes, con un nivel educacional alto, resulta preocupante los hallazgos que evidencian malos hábitos de vida, asociados a alta prevalencia de FRCV, las que son muy superiores a lo encontrado en jóvenes en población general a nivel nacional ${ }^{3}$.

$\mathrm{Al}$ analizar las variables metabólicas en la etapa de pre-intervención de la población en estudio, se encontró altas prevalencias de dislipidemia y glicemia alterada, especialmente en mujeres. Tras la intervención se logró disminuir tanto las preva- 
Intervención en factores de riesgo cardiovascular en estudiantes - A. M. Leiva et al

lencias de dislipidemia, como de glicemia alterada y a pesar que los resultados no son significativos para algunas de estas variables, se considera que un mayor tiempo de intervención podría asociase a mejores resultados.

Los altos niveles de sedentarismo encontrados en la etapa de pre-intervención concuerdan con otros estudios en población universitaria ${ }^{10-12}$. El principal motivo planteado por los estudiantes como impedimento para realizar actividad física en forma regular es "la falta de tiempo", lo que es coincidente con lo reportado en otras investigaciones $^{12,13}$.

La práctica de actividad física tiene un rol fundamental en la prevención, control y tratamiento de las ECV, por su influencia sobre varios $\mathrm{FRCV}^{14}$. En esta intervención, la incorporación obligatoria de dos sesiones de actividades deportivo-recreativas a la semana como requisito para aprobar la asignatura, permitió que $100 \%$ de los estudiantes categorizados como "sedentarios" en la etapa pre-intervención, aumentara la práctica de actividad física y $26,7 \%$ de este grupo pasara a la categoría de "activos", al asistir voluntariamente a una tercera sesión semanal. La incorporación de la actividad física en el contexto de una asignatura, permitió eliminar la barrera "falta de tiempo" aludida por los estudiantes, siendo esta estrategia un componente clave de la intervención.

Aunque esta intervención logró disminuir en $13 \%$ la prevalencia de exceso de peso (sobrepeso-obesidad), los resultados obtenidos en la etapa post-intervención, son superiores a lo reportado en estudio anterior realizado en estudiantes de primer año de la misma universidad ${ }^{6}$ y ratifica lo evidenciado por otros estudios a nivel nacional ${ }^{15,16}$, que indican que la prevalencia de exceso de peso aumenta con los años de universidad.

La transición desde la enseñanza media a la universidad representa un cambio drástico en los estilos de vida y conductas de los estudiantes asociadas al aumento de consumo de alcohol y tabaco, reducción de los niveles de actividad física y cambios en las conductas alimentarias ${ }^{17-20}$. Esto se evidencia a partir de este estudio realizado en estudiantes de tercer año, que encontró un alarmante aumento en todas las prevalencias de los principales FRCV, en comparación con investigación anterior realizada en estudiantes que ingresan a la universidad ${ }^{6}$.

Lo anterior, ratifica la necesidad de creación de programas permanentes, regulares y con créditos, que permita a los estudiantes universitarios incorporar en su vida personal un nuevo estilo de vida ${ }^{11}$.

Dentro de las conductas alimentarias, el consumo de frutas y verduras es un factor importante en la prevención de ECV y reducción de mortalidad asociada a estas enfermedades ${ }^{21}$. Un meta-análisis reciente sobre el consumo de estos alimentos reportó que los niveles de mortalidad disminuyen en $5 \%$ por cada porción de frutas y verduras consumidas al día ${ }^{21}$. A pesar de esta evidencia, en Chile, sólo $13 \%$ de los jóvenes consume 5 o más porciones de frutas y verduras al día, cantidad recomendada por la OMS ${ }^{1,3}$.

En este estudio, el consumo diario de frutas y verduras reportado en la etapa de pre-intervención es inferior a lo encontrado por la ENS 2009-2010 3 $\mathrm{y}$ otros estudios en población universitaria ${ }^{12,22}$, destacándose que ningún estudiante cumplía con las recomendación de 5 o más porciones diarias. Tras la intervención se logró que $20 \%$ de los estudiantes alcanzara dicha recomendación. El bajo consumo de frutas y verduras se puede asociar a que este estudio fue realizado en estudiantes universitarios del sur de Chile, zona que según lo publicado recientemente por la Encuesta Nacional de Consumo Alimentario, presenta el consumo más bajo de frutas y verduras a nivel país ${ }^{23}$.

En relación a las conductas asociadas al consumo de alcohol y tabaco, ambas se presentan con igual prevalencia en la etapa de pre-intervención en la población evaluada (50\%), siendo su modificación post-intervención no significativa. Esto revela la dificultad de cambiar en los jóvenes este tipo de conductas, que poseen un importante componente social al desarrollarse en compañía de amigos, durante eventos sociales y celebraciones. Ante este escenario, se visualiza la necesidad de incluir en las intervenciones programas multidisciplinarios de apoyo ${ }^{24}$.

Los resultados de esta intervención deberían considerarse dentro de las limitaciones asociadas al diseño del estudio, como la falta de un grupo control y el tamaño de la muestra que, al estar sujeta al cupo de una signatura, condicionó una distribución desigual de género, lo que puede haber producido un sesgo en la estimación de algunas prevalencias. Otra limitación del estudio fue el tiempo acotado de la intervención, al enmarcarse a un período académico.

La intervención desarrollada tuvo un impacto 
positivo en la reducción de los FRCV en los estudiantes evaluados, evidenciándose que la práctica regular de actividad física en el marco de un programa educativo de promoción de la salud y estilos de vida saludable, es una herramienta efectiva para la reducción de FRCV. La incorporación de estos contenidos en los planes de estudio desde los primeros niveles de formación, podría ser clave en la prevención y control de muchas enfermedades.

\section{Referencias}

1. World Health Organization. 2008-2013 Action plan for the global strategy for the prevention and control of noncommunicable diseases. WHO, Geneva, 2013. Disponible en: http://www.who.int/nmh/publications/ ncd_action_plan_en.pdf [Consultado el 7 de enero de 2014].

2. World Health Organization. Global health risks: Mortality and burden of disease attributable to selected major risks. WHO, Geneva, 2009. Disponible en: http://www. who.int/healthinfo/global_burden_disease/GlobalHealthRisks_report_full.pdf [Consultado el 14 de marzo de 2013].

3. Ministerio de Salud. Gobierno de Chile. Encuesta nacional de salud 2009-2010. MINSAL, 2010. Disponible en: http://web.minsal.cl/portal/url/item/bcb03d7bc28b64dfe040010165012d23.pdf [Consultado el 2 de abril de 2011].

4. Ministerio de Salud. Gobierno de Chile. Los objetivos sanitarios de la década 2000-2010. Evaluación final del período. Objetivos de impacto. MINSAL, 2010. Disponible en: http://epi.minsal.cl/epi/html/sdesalud/OS/EvaluacionObjetivosSanitarios2000-2010.pdf [Consultado el 2 de abril de 2011].

5. Escobar M. Prevención del riesgo cardiovascular: Políticas chilenas. Revista Médica Clínica Las Condes 2012; 23 (6): 651-5.

6. Martínez MA, Leiva AM, Sotomayor C, Victoriano T, Von Chrismar AM, Pineda S. Factores de riesgo cardiovascular en estudiantes de la Universidad Austral de Chile. Rev Med Chile 2012; 140 (4): 426-35.

7. Ministerio de Salud. Gobierno de Chile. Estrategia nacional sobre alcohol: reducción del consumo de riesgo y sus consecuencias sociales y sanitarias. Propuesta de un desafío bicentenario para Chile. MINSAL, 2010. Disponible en: file://C:/Users/M\%20Adela/Downloads/ Estrategia_Nacional_Alcohol_Chile_2010.pdf [Consultado el 15 de julio de 2013].

8. World Health Organization. Intervenciones eficaces en materia de régimen alimentario y actividad física. WHO,
Geneva, 2009. Disponible en: http://www.paho.org/hq/ index.php?option $=$ com_docman\&task=doc_view\&gi$\mathrm{d}=18318$ \&Itemid=2518\&lang=es [Consultado el 2 de abril de 2011].

9. Ministerio de Planificación (MIDEPLAN). Gobierno de Chile. Encuesta nacional de caracterización socioeconómica. CASEN 2011. Disponible en: http://observatorio. ministeriodesarrollosocial.gob.cl/casen_obj.php [Consultado el 7 de octubre de 2013].

10. MacMillan N. Valoración de hábitos de alimentación, actividad física y condición nutricional en estudiantes de la Pontificia Universidad Católica de Valparaíso. Rev Chil Nutr 2007; 34 (4): 330-6.

11. Rodríguez FJ, Espinoza LR, Gálvez J, MacMillan NG, Solís P. Estado nutricional y estilos de vida en estudiantes universitarios de la Pontificia Universidad Católica de Valparaíso. Universidad y Salud 2013; 15 (2): 123-35.

12. Olivares S, Lera L, Bustos N. Etapas del cambio, beneficios y barreras en actividad física y consumo de frutas y verduras en estudiantes universitarios de Santiago de Chile. Rev Chil Nutr 2008; 35 (1): 25-35.

13. Espinoza L, Rodríguez F, Gálvez J, MacMillan N. Hábitos de alimentación y actividad física en estudiantes universitarios. Rev Chil Nutr 2011; 38 (4): 458-65.

14. World Health Organization. Global recommendations on physical activity for health. WHO 2010. Disponible en: http://whqlibdoc.who.int/publications/2010/9789241599979_eng.pdf [Consultado el 15 de octubre de 2014].

15. Durán S, Castillo M, Vio F. Diferencias en la calidad de vida de estudiantes universitarios de diferente año de ingreso del campus Antumapu. Rev Chil Nutr 2009; 36 (3): 200-9.

16. Ratner R, Hernández P, Martel J, Atalah E. Calidad de la alimentación y estado nutricional en estudiantes universitarios de 11 regiones de Chile. Rev Med Chile 2012; 140 (12): 1571-9.

17. Bray SR, Born HA. Transition to university and vigorous physical activity: Implications for health and psychological well-being. J Am Coll Health 2004; 52 (4): 181-8.

18. Haase A, Steptoe A, Phil D, Sallis JF, Wardle J. Leisure-time physical activity in university students from 23 countries: associations with health beliefs, risk awareness, and national economic development. Prev Med 2004; 39 (1): 182-90.

19. Pullman AW, Masters RC, Zalot LC, Carde LE, Saraiva MM, Dan YY, et al. Effect of the transition from high school to university on anthropometric and lifestyle variables in males. Appl Physiol Nutr Metab 2009; 34: 162-71.

20. Kwan MY, Cairney J, Faulkner GE, Pullenayegum EE. 
Physical activity and other health-risk Behaviors during the transition into early adulthood: a longitudinal cohort study. Am J Prev Med 2012; 42 (1): 14-20.

21. Wang X, Ouyang Y, Liu J, Zhu M, Bao W, Hu FB. Fruit and vegetable consumption and mortality from all causes, cardiovascular disease, and cancer: systematic review and dose-response meta-analysis of prospective cohort studies. BMJ 2014; 349 (g4490): 1-14.

22. Mardones MA, Olivares S, Araneda J, Gómez N. Etapas del cambio relacionadas con el consumo de frutas y verduras, actividad física y control del peso en estudiantes universitarios chilenos. Arch Latinoam Nutr 2009; 59
(3): 304-9.

23. Ministerio de Salud. Gobierno de Chile. Encuesta nacional de consumo alimentario. Informe Final. MINSAL 2014. Disponible en: http://web.minsal.cl/sites/default/ files/ENCA-INFORME_FINAL.pdf [Consultado el 15 de diciembre de 2014].

24. Ministerio de Salud. Gobierno de Chile. Programa de Actividad Física para la prevención y control de los factores de riesgo cardiovasculares: documento MINSAL 2004. Disponible en: http://web.minsal.cl/portal/url/ item/75fe622727752266e04001011f0169d2.pdf [Consultado el 25 de noviembre de 2014]. 